\title{
Project Portfolio Selection under Uncertainty: A DEA Methodology using Predicted and Actual Frontiers
}

\author{
Ahmad Hassan \\ Research Associate, Operations Management and Information Systems \\ York University, Toronto, Canada, M3J 1P3 \\ E-mail: ahmadch.hassan@gmail.com
}

Wade Cook

Professor, Operations Management and Information Systems

York University, Toronto, Canada, M3J 1P3

E-mail:wcook@schulich.yorku.ca

Received: April 6, $2020 \quad$ Accepted: June 16, $2020 \quad$ Published: July 1, 2020

doi:10.5296/jmr.v12i3.17121 URL: https://doi.org/10.5296/jmr.v12i3.17121

\begin{abstract}
Project portfolio management (PPM) is an important area of interest in many organizations. There is a wide literature on each of many different aspects of PPM. The central purpose of the current paper is to focus on a specific sub-area of PPM, namely the project portfolio selection (PPS) problem. Specifically, we develop a new methodology that will aid management in choosing from a set of candidate project proposals, a subset of those project proposals that align with strategic objectives of the organization. Research methodology is based on the data envelopment analysis (DEA) construct to compare a set of decision making units (such as proposed projects) to arrive at an efficiency score for each member of this competing set, derive the best performers, generate an efficiency frontier and quantify inefficiency in the non-best performers. While DEA has been applied in numerous settings, the unique feature of the project portfolio application is the presence of two sets of data, namely pre-implementation "estimates", and post-implementation "actuals". Our methodology is unique in that it uses the idea of dual DEA frontiers based on such before and after data for a set of past projects. Dual frontier concept makes not only an important practical contribution to the PPS literature, but as well it opens new directions and provides an innovative advancement in the DEA literature. The requisite data is not publicly available. Therefore, we develop a general methodology to illustrate our technique.
\end{abstract}




\section{Macrothink

Keywords: Portfolio, DEA, Project Selection, Predicted Frontier, Actual Frontier 2020, Vol. 12, No. 3 


\section{Introduction}

Many organizations function in multi project environments when they have several projects in competition for resources, on-going simultaneously, and this is carried out through Project Portfolio Management. According to this management process, organizations should not manage the projects on an individual basis, but rather should observe them as unified assets of a single large portfolio to support and enable the realization of the organization's strategy (Levine, 2005). PPM is considered a dynamic decision-making process where a constant revision of the active development of projects take place. The evaluation, ranking and selection of new projects are carried out in this process. Decisions regarding reprioritization of the existing projects are made. The resources are allocated and reallocated accordingly (Cooper et al., 2001).

An important component in the management of projects is Project Portfolio Selection. The selection of the best composition of projects is a challenge as organizations maximize the effectiveness of a portfolio and calls for a systematic approach that considers the strategic objectives and constraints simultaneously. According to Project Management Institute (2015) Pulse of the Profession survey an emerging trend is the periodic benefit realization analysis of completed projects for strategy evaluation and organizational learning.

Finally, and central to the current paper, the predicted performance of a selected project and that project's eventual actual performance, should that project be approved and carried to completion, often do not agree. More to the point, it is often the case that pre estimates of project outcomes/benefits tend to be over stated. Therefore, project portfolios need to be evaluated by means of efficiency modeling that can translate the "pre" performance estimates into estimated "post" performance values.

In the current paper we focus on the portfolio selection problem. Specifically, we develop a methodology based on the data envelopment analysis (DEA) constructs of Charnes, Cooper and Rhodes (1978) also known as CCR Model that will aid management in making decisions based on the best information available. Unlike previous applications of the DEA concept to measure the relative efficiencies of a set of decision making units (DMUs), the portfolio selection problem can be viewed as one where the organization knows two things about a given "funded" project. First it knows what the "beliefs" were prior to carrying out the project, and second it knows what the realized outcomes were after the project was completed. Our proposed methodology makes use of past history on a set of projects where two sets of data are available.

\section{Background}

\subsection{Project Portfolio Management}

A Project Portfolio management framework is practiced through a set of processes, which collects data on consistent evaluation criteria across existing and proposed projects for the purposes of making relative comparisons (Souder, 1975). It is considered a dynamic decision making process and is conducted as a learning loop. The feedback and learning part from each completed project is intended to bring together any insights gained, involving a 
given set of past projects, which can be usefully applied to future projects. Then, pre and post analyses are carried out so that the actual performance of a project can be compared with initial predictions.

Unlike financial portfolios, there is a large literature gap in studying uncertainty in the context of project portfolios. This is because uncertainty has several dimensions and is difficult to manage because it relates to undesirable events that may or may not occur. In this research, performance uncertainty is studied in the context of proposed or predicted project benefits versus actual benefits that occur later. The benefits predicted for a project involve several qualitative and quantitative factors, and prediction errors can be observed upon completion of that project when the actual outcomes fail to match up with the predicted values. Hence, uncertainty also needs to be quantified in terms of the extent of benefits distribution that were achieved by comparable past projects and its variance in relation to the benefits mentioned in the proposed projects. Decision makers do not currently use such tools as proposed herein to appropriately measure project performance uncertainty when identifying project portfolios. This is part of the theoretical gap that the research herein attempts to address.

Substantial research has been carried out on the problem of dealing with uncertain data using the DEA methodology. Imprecise and fuzzy models are sometimes used to model uncertainty from a user input perspective and the external uncertainty perspective is dealt with by say stochastic models, by replacing deterministic data with random variables. To reiterate, what is different about the current problem setting is that there are two data sources to be dealt with: (1) for a set of past projects which were approved and carried through to completion, we consider the data on the proposers' perceived performance of those projects, and (2) data on the actual performance of those same (past) projects. Objectivity in this setting would be to make use of this data in a deterministic way. We go about this by considering two "frontiers" of best practice in the DEA sense, when evaluating comparable proposed projects for uncertainty. For one, this would allow for evaluating multiple criteria, which contribute to uncertainty, and secondly it can aid in reducing these measures to a single quantitative uncertainty measure.

\subsection{The Role of DEA}

One proposed methodology for selecting projects $j$ adopts the concept of an additive weighted total $z_{j}=\sum_{r} w_{r} y_{r j}$ of the scores $y_{r j}$ that each project $j$ is accorded on each of its criteria $r$. This is a framework that appears often in the literature, and the idea of using a linear weighted average or weighted total of output scores seems to be well understood and intuitively acceptable in the industry. The two issues to be addressed there are:

1. How to determine appropriate estimates of the criteria values $y_{r j}$ for a given proposed project, given that they are unknown in advance of actually implementing that proposed project; and 
2. How to set criteria weights $w_{r}$ so that the overall score $z_{j}$ can be estimated.

In the current paper, we adopt a related approach wherein we set out to derive an efficiency score for each proposed project. We later discuss the connection between these two methodologies.

The fact that projects being considered inevitably have multiple comparable measures, presents an opportunity to use the data envelopment analysis (DEA) methodology to aid in the selection of a project portfolio. As referenced above, DEA is a linear programming approach developed by Charnes et al. (1978) for measuring the relative performance efficiency. This technique can be used to measures how efficiently a DMU or project uses the resources available to generate a set of outputs. The outcome from applying the DEA methodology to a given set of comparable projects or DMUs, is the identification of a subset of the best performing or best practice members of that set, each of which is allocated a score of unity (1), and is deemed efficient. This subset of best practice projects generate what is called the efficient frontier. Each remaining (inefficient) DMU is assigned a score that reflects how far from the frontier that DMU actually is. This measure or score reflects the reduction in inputs or increase in outputs needed to bring that DMU to the frontier, thus rendering it efficient. In contrast to statistical methods such as regression analysis, that only give insights into average performing DMUs, DEA generates a piecewise empirical envelopment surface, which in economics terms represents the observed best practice efficiency frontier. Thus, as described above, the level of inefficiency of a decision making unit is determined by identifying improvements in inputs or outputs required to project that DMU onto the frontier.

To put things in context, we begin by presenting a version of the original Charnes et al. (1978) constant returns to scale (CRS) model. Suppose that in a general setting each member of a set of $n$ decision making units uses $m$ inputs to generate $s$ outputs. Each DMU "o" can now set out to either maximize its benefit to cost ratio, or minimize its cost to benefit ratio, depending on whether the organization wants to reduce inputs or increase outputs. Since we assume herein that it is output enhancement that the organization wishes to pursue, it is reasonable for that DMU to solve the following constrained fractional programming problem.

$$
\min Z_{o}=\frac{\sum_{i=1}^{m} v_{i} x_{i o}}{\sum_{r=1}^{s} u_{r} y_{r o}}
$$

Subject to:

$$
\frac{\sum_{i=1}^{m} v_{i} x_{i j}}{\sum_{r=1}^{s} u_{r} y_{r j}} \geq 1(j=1,2, \cdots, n)
$$


$v_{i} \geq 0, u_{r} \geq 0$

Here, $u_{r}$ and $v_{i}$ represent output and input multipliers, respectively. The constraints require that the ratio of the "virtual input" vs. "virtual output" for each $\mathrm{DMU}_{\mathrm{j}}$ should exceed 1. The objective is to obtain weights $\left(v_{i}\right)$ and $\left(u_{r}\right)$ that minimizes the ratio for DMU $U_{o}$, the DMU being evaluated. By virtue of the constraints, the optimal objective value $Z_{o}$ * is at least 1 . Charnes et al. (1978) show that the above fractional program (0) can be replaced by the following equivalent linear program (1)

$$
\min Z_{o}=\sum_{i=1}^{m} v_{i} x_{i o}
$$

Subject to:

$$
\begin{gathered}
\sum_{r=1}^{s} u_{r} y_{r o}=1 \\
\sum_{i=1}^{m} v_{i} x_{i j}-\sum_{r=1}^{s} u_{r} y_{r j} \geq 0, j=1, \ldots t, n
\end{gathered}
$$

$v_{i} \geq 0, u_{r} \geq 0$

As discussed in Charnes et al. (1978), the multipliers in (1) should in fact be transformed versions of those in model (0). However, with no loss of generality, we choose (for simplicity), to keep the same notation in the two models.

While model (1) does provide an efficiency score, it is often convenient to view efficiency by way of the dual of model (1), namely the linear model (2)

$$
\max \phi
$$

Subject to:

$$
\phi y_{r o}-\sum_{j=1}^{n} \lambda_{j} y_{r j} \leq 0, \forall r=1, \cdots, s
$$




$$
\sum_{j=1}^{n} \lambda_{j} x_{i j} \leq x_{i o}, \forall i=1, \cdots, m
$$

\section{$\lambda_{j} \geq 0, \phi$ unrestricted in sign}

The variable $\phi$ measures the enhancement factor for the outputs. Specifically, the CCR model is referred to as a radial projection model, this name being derived from the fact that outputs are proportionally increased by an amount $\phi$; this factor measures the amount by which outputs must be "scaled up" along a ray projected through the origin, such as to move or "project" the DMU to the frontier. The $\lambda$ variables identify which efficient DMUs on the frontier are the ones against which the DMU in question is being compared.

In the DEA literature model (1) is generally referred to as the dual linear model or multiplier model, and model (2) as the primal or envelopment model.

The original DEA model of Charnes et al. (1978) used the constant returns to scale (CRS) technology, which means that a proportional increase in inputs results in a proportionate increase in outputs. While the CRS may be appropriate in many settings, in other cases this may not reflect reality, and it is the variable returns to scale (VRS) model, introduced by Banker et al. (1984), denoted as BCC, that is more appropriate. The difference between the two types of envelopment surfaces, CRS and VRS, is the presence of a convexity constraint $\sum_{j=1}^{n} \lambda_{j}=1$ in the case of VRS.

Due to the convexity constraint in the VRS model, DEA projections are always bounded within the observed values. For example, if all project performance measures are rated on a scale from say 0 to 100 , then during the evaluation process one should not exceed 100 $\%$. In the general DEA structure with multiple inputs and outputs, this condition can be violated under the constant return to scale model due to the ray expansion. The VRS technology, however, forms a convex hull of intersecting planes which envelope the data points more tightly than the CRS canonical hull, thus providing technical efficiency scores that are equal to or greater than those obtainable by means of the CRS model. The BCC model also distinguishes the scale in which a DMU operates as displaying increasing, decreasing or constant returns to scale, making the CCR model a special case of the BCC model.

\section{Methodology}

\subsection{Project Ranking versus Portfolio Construction}

Before proceeding it is important to point out that construction of a project portfolio involves the creation of a mix of different forms of projects, e.g. projects with large budgets 
and those with small budgets, projects with short and others with long development periods, etc. Thus, one can view portfolio construction as a process of creating a mix of projects from a set of "buckets" of proposed (and existing) initiatives. It is understood that within any bucket, projects are similar in "size" and scope. We view this as a two-stage process. The first stage entails ranking the projects inside each bucket, utilizing the fact that two data sets are involved (pre and post data). Stage 2 utilizes these multiple rankings to select a best portfolio. In the current paper we focus attention on the first stage, as the second stage involves many considerations beyond the scope herein. Some of these considerations may involve intra as well as inter bucket considerations. Specifically, there can be connections and inter dependencies between projects in one bucket and those in other buckets; e.g. certain projects may be undertaken only as part of a package of projects across buckets.

\subsection{Ranking Project Proposals within a Bucket}

Ranking a set of proposed projects involves two sets of activities. The first set of activities involves developing an efficient frontier at both the pre-implementation and post-implementation levels. The second set of activities concerns the use of those frontiers for each member of the set of proposed projects to generate predicted output bundles, predicted efficiency scores, and finally a ranking of the projects within each bucket.

Due to non-availability of real data, a general methodology has been developed to demonstrate our technique. The requisite data to implement this methodology would generally be available only to management in the organization involved.

\subsection{Proposed Three Steps Methodology}

As discussed above, our proposed methodology for selecting projects $j$ adopts the output oriented VRS DEA model of Banker et al. (1984), namely model (2) together with the appropriate convexity constraint $\sum_{j=1}^{n} \lambda_{j}=1$. We apply this methodology using both a pre-implementation (or predicted) best practice frontier, and a post-implementation (actual) frontier. As discussed, these frontiers would be based on pre and post criteria scores of past projects, obtained from internal records. The methodology involves three steps.

\subsubsection{Step 1: Establishing the Pre and Post Frontiers}

Let us suppose the organization selects a set of past project proposals that were ultimately carried through to completion. We assume that for each of these projects two sets of data are available, namely predicted outcomes (as predicted by the proposer of the project prior to the project being selected), and the actual outcomes that resulted when the project was implemented and ultimately completed. For discussion purposes it is assumed that the data consists of outputs or outcomes measured on Likert scales. For illustrative purposes we assume there are six outputs and that a 5-point Likert scale is used. Figure 1 is an example of a project rating spreadsheet method taken from (Pinto, 2010). 


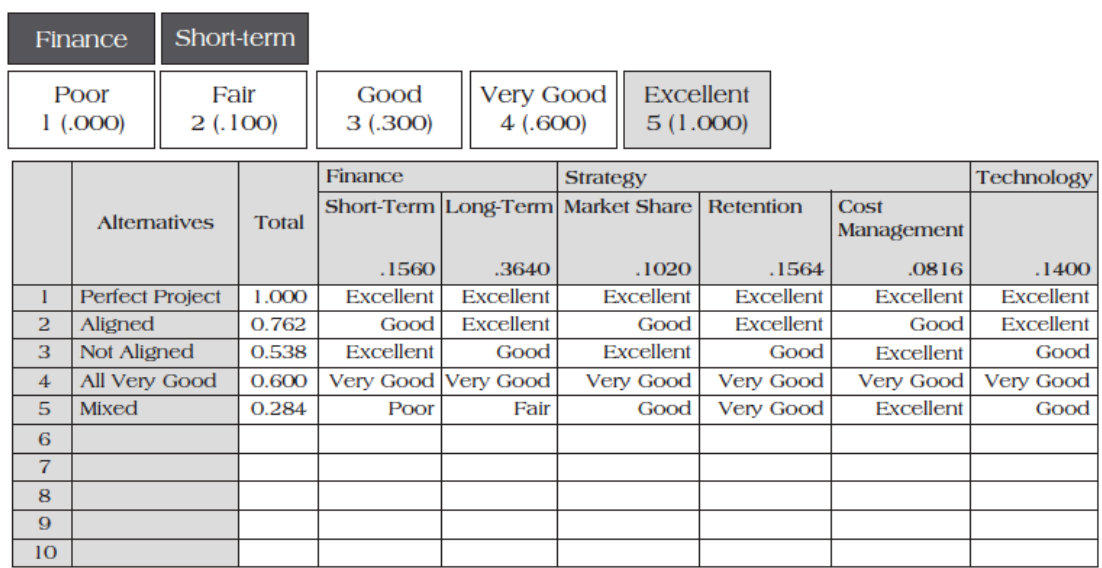

Figure 1. Sample Project Rating Spreadsheet

There, each project is rated, from an output perspective, on six management-defined criteria, and in that particular case a total "score" is calculated via a weighted total of the outcomes on the chosen criteria. Our proposed model compliments this methodology. In our model all performance measures follow "higher the better" rule, otherwise known as the profit characteristic, to produce the project's figure of merit. For a profit characteristic, increasing is the direction for improvement. From a DEA perspective, this means that DMUs are evaluated using outputs only; no input measures are involved in these types of data sets. While the pure output approach is consistent with a number of previous studies, we wish, at the same time, to adopt the DEA methodology. To make the DEA method operational, we assume there to be only one input, namely "1" for each project (Du et al., 2012).

While the problem herein is constructed on the presumption that all considered data are measured on Likert scales and represent outputs, there are practical situations where one needs to consider the presence of ratio scale inputs such as a budget. To handle such data in the model structure discussed below, a recommended approach is to treat such factors as undesirable outputs. In simple terms one can replace a positive input $x$, such as portfolio budget, by the negative of that factor $\hat{x}=-x$, thereby replacing a positive input by a negative "undesirable output". To create a "desirable output" one can then set some form of upper limit $x_{\max }$ (say the maximum budget one might encounter) and replace $-x$ by $x^{*}=x_{\max }-x$.

Using the two data sets for the selected projects (pre and post data), a DEA analysis is conducted on each, applying the VRS version of model (3). Once the two DEA analyses have been done, we keep only the efficient DMUs from each set, namely we retain the subset of DMUs P that turn out to be efficient when model (3) is applied to the predicted data, and the subset of DMUs A that are efficient under model (3) when applied to the actual outcomes for those projects. These two sets of efficient units define the pre and post frontiers. 
3.3.2 Step 2: Evaluating New Proposed Projects Relative to the Predicted Frontier

As discussed above, DEA defines DMUs as efficient when they lie on the frontier. Unlike the inefficient units, the efficient ones cannot easily be further classified based on their efficiencies that arise from a model such as (3), due to the fact that those efficient units all have the same efficiency score of unity. It is not, however, reasonable to simply claim that the efficient DMUs all have the same performance in actual practice. A number of approaches have been suggested for prioritizing the efficient units, with one such methodology being the super efficiency (SE) approach proposed in the seminal work of (Andersen et al., 1993). Their approach involves applying model (2), but with the DMU under evaluation not included in the reference set of the standard DEA model. The super efficiency model in the output-oriented case is that given by (in its envelopment form) (3). Notice that in model (3), unlike in (2), the DMU "o" under consideration is not included in the reference set. This is a case of the output oriented radial-projection VRS DEA model.

$\max \phi$

Subject to

$$
\begin{gathered}
\phi y_{r o}-\sum_{j=1, j \neq o}^{n} \lambda_{j} y_{r j} \leq 0, \forall r=1, \cdots, s \\
\sum_{j=1, j \neq 0}^{n} \lambda_{j} x_{i j} \leq x_{i o}, \forall i=1, \cdots, m \\
\sum_{j=1}^{n} \lambda_{j}=1
\end{gathered}
$$

$\lambda_{j} \geq 0, \phi$ unrestricted in sign

The efficient units can be ranked further by computing their super efficiency scores. An output-oriented efficiency score less than unity can result from the (output oriented) super efficiency model. For example, if DMU B is not included, the new efficient frontier will be formed using only DMUs A and C. B's distance to the new frontier will then be calculated in order to ascertain its super-efficient score. The added or incremental efficiency $1-\phi^{*}$ provides the allowable decrease in its outputs before it (B) would become inefficient. The super-efficient DMU enjoys output gains. The output gain of the super-efficient DMU $\mathrm{B}$ is $\left(1-\phi^{*}\right) Y_{B}$; e.g. if the super efficiency score for $\mathrm{B}$ is 0.9 , this means that $\mathrm{B}$ 's outputs can decrease by 10 percent and still stay efficient.

To be clear, the original idea behind the SE measure for an efficient DMU, was to have a score that would serve two purposes. One purpose was to provide a "stability" region or zone within which the output bundle for that DMU could be decreased, while still 
maintaining the efficient status of that DMU. This is a useful concept in that it provides a type of uncertainty region within which outputs can be altered. The second purpose of the SE score was to provide a means whereby the set of efficient units could be ranked. In both of these situations the DMU being investigated is "removed from the reference set".

In the present situation we use the SE measure for a reason somewhat different from those described in the previous paragraph. The frontier of best performance (based on say data in the form of perceived outcomes) is first established using past implemented projects. Then, keeping the frontier fixed, a new proposed project is evaluated against that fixed frontier. As will be discussed below, it will be a form of the super efficiency model that will be applied, rather than the conventional model (2).

When a new project is proposed, it is of value to the organization to evaluate the claimed or expected future performance of that project, from the perspective of the proposer, against the best past practice predicted frontier. Namely, the proposed project should be evaluated using the set of efficient projects $\mathrm{P}$ and applying the super efficiency model (3). If the efficiency score $\phi$ is equal to unity, then the proposed project lies on the 'predicted

frontier. If $\phi$ is greater than unity, then it is inefficient relative to the predicted frontier. If $\phi$ is less than unity, then the proposed project would be declared as being super-efficient.

\subsubsection{Step 3: Estimating the Performance of Proposed Projects using the Actual Frontier}

Since the actual outcomes of a proposed project, should it be chosen for implementation, are unknown, we suggest using the history linking predicted and actual performance, in the form of the two frontiers. Let us suppose that a new project (pre-implementation) is predicted by the proposer to perform according to the point $R_{o}$. What we wish to do is use $R_{o}$ to make an inference as to what the actual (post-implementation) score $\hat{R}$ might be.

We argue that given a point $R_{o}$, and its efficiency score $\phi_{R_{o} P}$ relative to the predicted frontier, it is reasonable to argue that the "expected" actual point $\hat{R}$ will be such that its estimated efficiency score $\hat{\phi}_{\hat{R} A}$ against the actual frontier should be related to $\phi_{R P}$, or be expressed in the form of some function of $\phi_{R P}$ as shown in Figure 2. That is, we wish to find a point $\hat{R}$ such that $\hat{\phi}_{\hat{R} A}=f\left(\phi_{R_{o} P}\right)$. Such a function $f$ might reasonably take the form of a simple linear regression model

$$
\hat{\phi}_{\hat{R} A}=a+b \phi_{R_{o} P}
$$




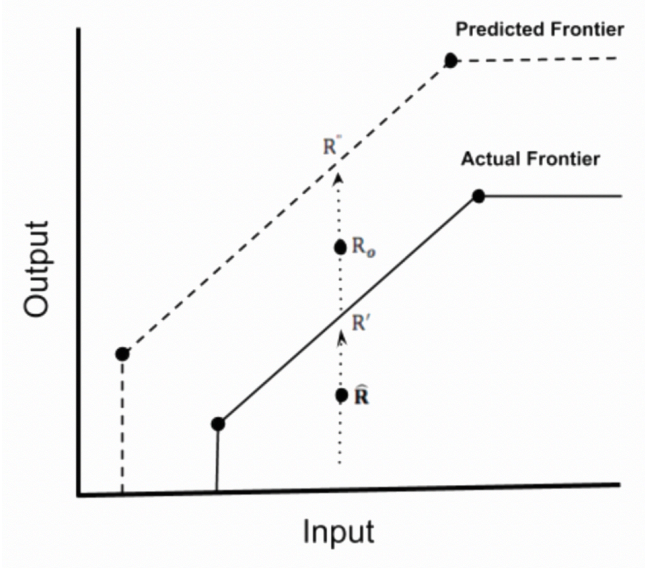

Figure 2. Estimated $R_{o}$ projections

If the predicted frontier overlooks the actual frontier, then let $R^{\prime}$ denote the projection of $R_{o}$ onto the actual frontier, that is

$$
R^{\prime}=R_{o} \phi_{R_{o} A}
$$

As well, we also have

$$
R^{\prime}=\hat{R} \hat{\phi}_{\hat{R} A}
$$

But, since we assume $\hat{\phi}_{\hat{R} A}=a+b \phi_{R_{o} P}$ as per (4) above, it follows from (5) and (6) that

$$
\begin{array}{r}
\hat{R} \hat{\phi}_{\hat{R} A}=R_{o} \phi_{R_{o} A} \text {, meaning that } \hat{R}\left(a+b \phi_{R_{o} P}\right)=R_{o} \phi_{R_{o} A} \text {, or } \\
\hat{R}=R_{o} \phi_{R_{o} A} /\left(a+b \phi_{R_{o} P}\right)
\end{array}
$$

Hence, the estimated post-implementation output bundle $\hat{R}$ becomes the adjusted version of the pre-implementation predicted point $R_{o}$.

A somewhat more realistic setup is the possibility that the two frontiers may cross each other. This phenomenon can happen when for some proposed projects, there may be a tendency to overstate outcomes for certain criteria, while understating outcomes in the case of other criteria. We refer to the situation where the predicted frontier is above the actual frontier as Case 1. Case 2 is the situation where the actual frontier dominates the predicted frontier.

Case 1: We start with the assumption that the predicted frontier is higher than (dominates) the actual frontier. Again, as in the single output case discussed above, let $R_{o}$ represent the 


\section{Macrothink}

predicted performance of the project in question, prior to implementation, and let $R^{\prime}$ represent the projected version of $R_{o}$ onto the actual frontier. Using the same argument as in the single output case, we express $R^{\prime}$ in two forms, namely $R^{\prime}=R_{o} \phi_{R_{o} A}$ and $R^{\prime}=\hat{R} \hat{\phi}_{\hat{R} A}$. Again, we postulate that $\hat{\phi}_{\hat{R} A}=a+b \phi_{R_{o} P}$ as in (4) above. As earlier, $\hat{R} \hat{\phi}_{\hat{R} A}=R_{o} \phi_{R_{o} A}$, meaning that $\hat{R}=R_{o} \phi_{R_{o} A} / \hat{\phi}_{\hat{R} A}$ or $\hat{R}=R_{o} \phi_{R_{o} A} /\left(a+b \phi_{R_{0} P}\right)$, as in (7).

Case 2: This is the situation where the actual frontier dominates the predicted frontier. The same arguments as those given for Case 1, lead to the same conclusion, namely that the adjusted profile of criteria scores is given by (7).

In the general multiple output setting where the frontier is known, one would normally apply model (3) to derive an efficiency score $\phi_{j_{o}}$, provided the outputs $y_{r j}$ are known quantities. In our case, however, if the efficiency score $\hat{\phi}_{\hat{R} A}$ has been estimated, it is the actual (post-implementation) output bundle $\hat{R}$ that we wish to determine. Consider the following model (8).

$$
\widehat{T}=\max T
$$

Subject to

$$
\begin{gathered}
\hat{\phi}_{\hat{R} A} y_{r o}-\sum_{j=1, j \neq o}^{n} \lambda_{j} y_{r j} \leq 0, \forall r=1, \cdots, s \\
\sum_{j=1, j \neq o}^{n} \lambda_{j} x_{i j} \leq x_{i o}, \forall i=1, \cdots, m \\
y_{r o} \geq T \bar{y}_{r o}, \forall r=1, \cdots, s \\
\sum_{j=1 j \neq o}^{n} \lambda_{j}=1 \\
\lambda_{j} \mathrm{~T} \geq 0, \hat{\phi}_{\hat{R} A} \text { unrestricted in sign }
\end{gathered}
$$

In (8), the members of the output vector $\hat{R}=\left(y_{r o}\right)$ constitute the decision variables. We propose to have the vector $\hat{R}$ proportional to $R_{o}$, whose components are given by $\bar{y}_{\text {ro }}$. That is, we wish to derive components $\hat{R}=\left(y_{r o}\right)$ as scaled versions of those in $R_{o}$. Constraints (8.4) are meant to represent that derivation. The scaling factor is the decision variable $\mathrm{T}$ whose optimal value is given by $\hat{T}$. In the development above the focus centered on arriving 
at an estimated profile $\hat{R}$ that represents the organization's best guess as to how the proposed project will perform post-implementation.

\section{Concluding Remarks}

In the present study we have developed a methodology for aiding in the selection of projects in a project portfolio setting. In such environments it is assumed that each project will be evaluated in terms of a set of criteria measured on Likert scales. It is assumed as well that two sets of data on a set of past projects are available to management, namely (1) data relating to predictions about how those projects were estimated (at the time they were proposed) to perform if implemented, and (2) data revealing how those projects actually performed when finally carried through to completion. The argument is that this pre and post historical record can provide a means of transforming pre-implementation predictions of any proposed project into estimated actuals. Our suggested approach to selecting a portfolio of projects from a given bucket is to apply the DEA model to each of these two sets of data, thereby generating pre and post frontiers. These two frontiers can then be used to adjust pre-implementation predictions to reflect known historical differences between estimated and actual values. It is recommended that the pre and post frontiers be updated on a regular basis.

It is instructive to point out that there is a connection between the DEA methodology presented herein, versus the general approach in the literature involving deriving an additive weighted total $z_{j}=\sum_{r} w_{r} y_{r j}$ of the scores $y_{r j}$ that each project $j$. Specifically, in the presence of a single input, set at unity, model (0) becomes one of minimizing $1 /\left(\sum_{r=1}^{S} u_{r} y_{r j}\right)$, or equivalently, maximizing its inverse $\sum_{r=1}^{S} u_{r} y_{r j}$. The difference between the two approaches lies in the fact that model (0) seeks a best set of multipliers, for a given project, in the presence of a set of previous proposals. Furthermore, these multipliers may differ from one project to another. Hence, our approach acknowledges the fact that multiplier choice is subjective, and offers a systematic approach to implementing this choice.

\section{References}

Andersen, P., \& Petersen, N. C. (1993). A Procedure for Ranking Efficient Units in Data Envelopment Analysis. Management Science, 39(10), 1261-1264. https://doi.org/10.1287/mnsc.39.10.1261

Banker, R. D., Charnes, A., \& Cooper, W. W. (1984). Some Models for Estimating Technical and Scale Inefficiencies in Data Envelopment Analysis. Management Science, 30(9), 1078-1092. https://doi.org/10.1287/mnsc.30.9.1078

Charnes, A., Cooper, W. W., \& Rhodes, E. (1978). Measuring the efficiency of decision making units. European Journal of Operational Research, 2(6), 429-444. https://doi.org/10.1016/0377-2217(78)90138-8 


\section{Macrothink $\Lambda$ Institute}

Cooper, R. G., Edgett, S. J., \& Kleinschmidt, E. J. (2002). Portfolio Management For New Products: Second Edition (2nd ed.). Basic Books.

Du, J., Chen, C.-M., Chen, Y., Cook, W. D., \& Zhu, J. (2012). Additive super-efficiency in integer-valued data envelopment analysis. European Journal of Operational Research, 218(1), 186-192. https://doi.org/10.1016/j.ejor.2011.10.023

Levine, H. A. (2005). Project Portfolio Management: A Practical Guide to Selecting Projects, Managing Portfolios, and Maximizing Benefits (1st ed.). Jossey-Bass.

Pinto, J. K. (2010). Project management: Achieving competitive advantage (2 ${ }^{\text {nd }}$ edition). Pearson/Prentice Hall.

PMI. (2015). Capturing the Value of Project Management. Retrieved from https://www.pmi.org/learning/thought-leadership/pulse/capturing-the-value-of-project-ma nagement

Souder, W. E. (1975). Achieving Organizational Consensus with Respect to R\&D Project Selection Criteria. Management Science, 21(6), 669-681. https://doi.org/10.1287/mnsc.21.6.669

Acknowledgement: This research was supported by the National Sciences and Engineering Research Council Canada, grant number A8966. 